\title{
Portugol Studio: Em direção a uma comunidade aberta para pesquisa sobre o aprendizado de programação
}

\author{
Adson Marques da Silva Esteves, Luiz Fernando Noschang, André Luis Alice \\ Raabe, Aluizio Haendchen Filho
Mestrado em Computação - Universidade Do Vale do Itajaí (UNIVALI) - Campus de Itajaí
88032-005 - Itajaí - SC- Brasil
shinadson@gmail.com, noschang@univali.br, raabe@univali.br, aluizio.h.filho@gmail.com

\begin{abstract}
The goal of this paper is to show the advances of the IDE didactic Portugol Studio (PS) in these last 5 years detailing the improvements made in the newer versions, the enhancements focused in expanding the usability of the tool, video lessons, didactic material produced by its users, emphasize the interfaces for the development of plugins, the produced works and show the users profiles the platform attends. Lastly to invite the community to develop new plugins and evolve PS.
\end{abstract}

Resumo. O objetivo deste artigo é apresentar os avanços da IDE didática Portugol Studio (PS) nos últimos 5 anos detalhando as melhorias realizadas nas novas versões, os aprimoramentos focados em ampliar a usabilidade da ferramenta, videoaulas e materiais produzidos pelos seus usuários, enfatizar as interfaces para o desenvolvimento de plugins, os trabalhos já produzidos e apresentar o perfil dos usuários que a plataforma atende. Por fim convidar a comunidade a desenvolver novos plugins e evoluir o PS.

\section{Introdução}

A aprendizagem de programação no Brasil enfrenta diversos problemas, como já foi analisado em outros trabalhos realizados na área. Noschang et al, 2014 faz uma análise de alguns destes trabalhos e aponta como principais barreiras o uso do idioma Inglês e a complexidade das ferramentas de desenvolvimento (IDEs) existentes.

Segundo os autores, a maioria da IDEs não é adequada aos estudantes iniciantes por apresentarem as seguintes características: (i) linguagem de programação e textos da interface gráfica no idioma inglês; (ii) quantidade elevada de elementos na interface gráfica (menus, configurações, janelas) que intimidam o usuário; (iii) mensagens de erro em Inglês e de difícil compreensão, muitas vezes confusas; (iv) complexidade no uso e configuração do depurador e; (v) sintaxe das linguagens é complexa.

Dentre deste contexto, o Portugol Studio é um ambiente de desenvolvimento que foi criado com o enfoque na facilitação da aprendizagem de programação para usuários que dominam o idioma da língua portuguesa. Esse ambiente foi desenvolvido com o propósito de reduzir as dificuldades que iniciantes em programação passam ao se depararem a primeira vez com as linguagens de programação e ambientes de desenvolvimento comerciais. 
O Portugol Studio foi desenvolvido na linguagem Java e divide-se em três módulos principais: (i) núcleo, responsável por processar e executar a linguagem Portugol; (ii) IDE, representa a interface gŕaifca com suas funcionalidades e componentes e consome os recursos disponibilizados pelo núcleo e; (iii) console, responsável por permitir a execução de programas em portugol, através do console do sistema operacional. A figura 1 apresenta a arquitetura simplificada do Portugol Studio.

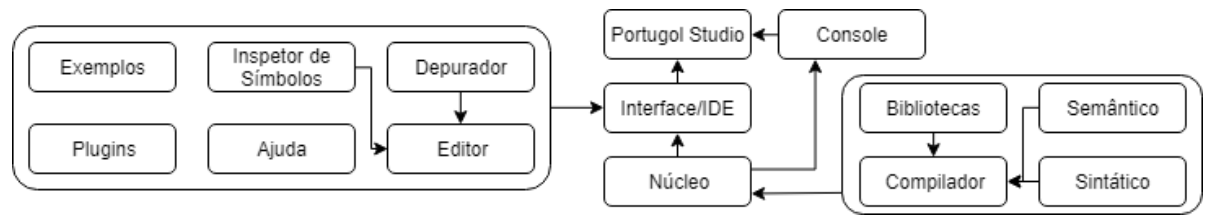

Figura 1. Arquitetura simplificada do Portugol Studio

A plataforma foi lançada em 2013 dentro de grupos acadêmicos, atingindo 2000 downloads em oito meses (Noschang et al, 2014). Em 2014 um artigo submetido ao XXII Workshop sobre Educação em Computação - WEI foi selecionado entre os melhores artigos publicados. Atualmente, conta com mais de 240.000 downloads em todo o Brasil e países de língua portuguesa. Desde seu lançamento, muitas mudanças ocorreram na plataforma. Seu layout foi melhorado e modernizado, houveram melhorias de desempenho, foram adicionadas novas bibliotecas, novos exemplos, inspetores para depuração e a possibilidade de adicionar plugins à ferramenta.

Este artigo tem como objetivo relatar as principais mudanças e melhoramentos que ocorreram no Portugol Studio durante os últimos cinco anos de desenvolvimento. $O$ principal foco será enfatizar as melhorias realizadas na ferramenta, suas diretrizes e impactos, o perfil dos usuários e o envolvimento da comunidade.

\section{Melhorias implementadas}

A primeira versão do Portugol Studio começou a ser utilizada em 2011 dentro da UNIVALI (Universidade do Vale do Itajaí), instituição do Laboratório de Inovação Tecnológica na Educação (LITE) onde a plataforma foi criada por Luiz Fernando Noschang, graduando de ciência da computação na época. Dois anos depois a ferramenta chegou na sua versão 2.0, começando a ser utilizada em outras universidades como a Universidade Federal do ABC e um ano depois alcançou a versão 2.2.

Nas seguintes subseções será mostrada a evolução do Portugol Studio até a versão atual, como também quais os novos recursos que a ferramenta obteve desde seu último relato por Noschang em 2014.

\subsection{Lançamentos}

O Portugol Studio passou por diversas atualizações durante esses cinco anos. A seguinte tabela apresenta todas as versões que foram lançadas até o momento. 
Tabela 1. Versões lançadas do Portugol Studio

\begin{tabular}{|l|l|l|}
\hline Versão & Data & Principais adições \\
\hline v1.0 & $30 / 11 / 2011$ & Interpretador; Depurador; Exemplos; Bibliotecas; \\
\hline v2.0 & $19 / 08 / 2013$ & Ajuda; Temas para o código; \\
\hline v2.2 & $19 / 06 / 2014$ & Video-aulas; Mecanismo de plugins; \\
\hline v2.5 & $15 / 04 / 2016$ & Novo Layout; Pontos de parada; Inspetor; \\
\hline v2.6 & $07 / 06 / 2017$ & Temas para interface; Arquivos recentes; Recuperação; Reporte no Github; \\
\hline v2.6.1 & $13 / 09 / 2017$ & Problemas gerais resolvidos; \\
\hline v2.6.2 & $04 / 09 / 2017$ & Captura de exceções para reporte; \\
\hline v2.6.3 & $06 / 10 / 2017$ & Notificador de atualização; \\
\hline v2.6.4 & $10 / 11 / 2017$ & Biblioteca de Calendário; Alterações nos exemplos; \\
\hline v2.6.5 & $15 / 12 / 2017$ & Indentador automático; \\
\hline v2.6.6 & $02 / 03 / 2018$ & Biblioteca de Internet; \\
\hline v2.6.7 & $06 / 04 / 2018$ & Layout da tela inicial; Ajuda unificada; Bibliotecas Objetos e Serviços Web; \\
\hline v2.6.8 & $13 / 04 / 2018$ & Auto-Complete; \\
\hline v2.6.9 & $14 / 05 / 2018$ & Função sorteia como parte da linguagem; \\
\hline v2.7.0 & $02 / 11 / 2018$ & Plugins refeitos; Customizador de tema; \\
\hline v2.7.1 & $21 / 12 / 2018$ & Alteração do charset pra UTF-8; \\
\hline v2.7.2 & $22 / 03 / 2019$ & Templates de código no Auto-Complete; \\
\hline
\end{tabular}

\subsection{Novos recursos}

Nas seguintes subseções são apresentados alguns dos principais recursos novos do Portugol Studio, buscando mostrar seus objetivos e vantagens. Para o recurso dos plugins a seção 3 será utilizada para uma explicação completa e exemplificação de trabalhos com ela.

\subsubsection{Pontos de parada}

A versão 2.5 do Portugol Studio trouxe o recurso Pontos de parada. Este recurso permite que em um trecho de código com comandos, um "inseto" ao lado esquerdo da linha seja ativado, criando uma flag. Quando o usuário executa normalmente o seu código, o modo de depuração será ativado automaticamente assim que a linha com a flag adicionada for ser executada, facilitando a depuração de funções e trechos específicos do código.

$\mathrm{Na}$ versão anterior, o usuário que quisesse depurar seu código precisava passar por todas as instruções até chegar na parte que era necessária a ele, fazendo com que em programas grandes, um grande tempo fosse perdido desnecessariamente.

\subsubsection{Inspetor de símbolos}

Também na versão 2.5 foi adicionado o Inspetor de símbolos. Esse recurso existe no canto inferior direito do Portugol Studio abaixo da árvore de símbolos. Ele permite que os valores das variáveis adicionadas a ele, possam ser visualizados em tempo real durante a execução do código. Essas variáveis podem ser adicionadas a ele ao arrastá-las tanto do código quanto da árvore para a área do inspetor.

O recurso evita que o usuário durante a depuração utilize funções como o escreva() para descobrir o valor de alguma variável durante a depuração, assim deixando o console limpo para saídas próprias do programa. 


\subsubsection{Novas Bibliotecas}

Durante estes 5 anos bibliotecas foram adicionadas e modificadas. As atualizações contavam com novas funções para bibliotecas já existentes permitindo o usuário manipular mais informações de seus tipos de arquivos respectivos. Já nas bibliotecas novas, possibilidades de criação de objetos e conexão com a internet também foram possíveis.

\subsubsection{Temas}

Junto com uma mudança de layout do Portugol Studio na versão 2.6, foi verificado quais cores seriam mais apropriadas para uma IDE. A maioria variava entre cores claras com o fundo do código fonte branco e entre cores escuras. Foi percebido que as IDEs mais recentes utilizavam cores escuras como padrão ou opcionais, enquanto as mais antigas utilizavam um padrão claro, sem muitas opções de troca de cores.

Após professores, alunos e usuários independentes trazerem opiniões divergentes sobre cores que poderiam ser utilizadas na IDE, foi decidido dar o poder de selecionar as cores da plataforma ao usuário. Na versão 2.7, foi lançado um Editor de Temas, ele contém 2 temas básicos, o tema claro e escuro, porém o usuário pode adicionar novos temas, podendo se adaptar as cores que o usuário achar melhor.

\section{Suporte a plugins}

A plataforma Portugol Studio, possibilita adicionar plugins externos a ele. Os plugins são programas em java que tem acesso às funções internas do PS. Permite que o desenvolvedor do plugin possa fazer alterações em qualquer interface, comportamento ou extrair informações de uso, como o código fonte que o usuário cria.

Por serem plugins, eles não vêm pré-instalados no Portugol Studio, sendo necessário que os usuários que queiram utilizar plugins já desenvolvidos baixem eles separadamente. Pensando nesse caso, dentro da plataforma há um agregador de plugins desenvolvidos com links para seus repositórios, além da possibilidade de baixar eles na própria plataforma.

Para plugins desenvolvidos serem adicionados a esse agregador, é necessário o desenvolvedor contatar a equipe do Portugol (através do reporte de bugs é possível ver formas de contato). Dessa forma é possível avaliar se o plugin está pronto para ser adicionado ao agregador e guiar o desenvolvedor para montar um repositório e um release para ser adicionado à plataforma.

Nas seguintes subseções serão apresentados alguns plugins desenvolvidos por alunos de Trabalhos de Conclusão de Curso como também Dissertações de Mestrado. Atualmente apenas o plugin de GoGo Board está adicionado ao agregador por estar funcional, sendo que os outros ainda estão em fase de testes e correções. Na última subseção será explicado o esforço necessário para se criar um plugin.

\subsection{GoGo Board}

Cardoso (2017) desenvolveu o plugin de GoGo Board para o Portugol Studio. A GoGo Board é uma placa de baixo custo e código aberto, para robótica e experimentação educacional [Blikstein 2004]. O plugin foi desenvolvido buscando uma aproximação da programação para iniciantes e a robótica dentro das salas de aula [Cardoso 2017]. 
O plugin foi baseado na IDE de programação oficial da Gogo Board, o GoGo Widget. Tem uma interface de controle das funcionalidades da placa através de botões, como também a possibilidade de enviar códigos programados em portugol para ela. Também é possível através das bibliotecas que estão no plugin, executar programas diretamente na IDE que mandem ou recebam informações da placa durante a execução, permitindo que softwares em portugol interajam com a GoGo Board em tempo real.

\subsection{Simulador}

Breda (2017) desenvolveu um plugin de simulação de experimentos para o Portugol Studio. Trazendo as ideias de modelamento bifocal, o trabalho trouxe uma biblioteca que permite criar agentes e dar ações a eles, similar ao NetLogo.

O modelamento bifocal tem como objetivo ligar os modelos computacionais com a coleta de dados prática no mundo físico. Através da análise entre o modelo computacional e o experimento físico pode se compreender melhor como o fenômeno ocorre [Blikstein 2014].

O plugin permite que a programação em portugol, possa ser utilizada para dar funções aos agentes, mesmo a linguagem não tendo conceito de agentes ou objetos. Para se visualizar a simulação, um ambiente gráfico dentro do plugin é utilizado, onde são mostrados os agentes e as variáveis do código, similar a interface do Netlogo. Ainda está em fase de ajustes, necessitando passar por testes para a identificação e correção de problemas.

\subsection{Criador de Aplicativos}

Foi desenvolvido um plugin de criação de aplicativos para o sistema operacional Android. O plugin traduz e compila a programação em portugol que o usuário faz em linguagem bitcode de LLVM e instala a aplicação no smartphone android que estiver conectado no computador. Além disso, ele trás consigo um controle de videogame com setas, para controlar as ações das aplicações criadas no smartphone.

O bitcode de LLVM é como uma linguagem intermediária entre o código portugol e o código nativo do dispositivo, no caso, java para android. O plugin ainda não está completo, sendo buscado adicionar outras formas de controle a ele, além de correções de problemas.

\subsection{Criação de um Plugin}

Para o desenvolvimento de um plugin para o Portugol Studio, a equipe disponibilizou um plugin de exemplo em um repositório aberto separado ${ }^{[1]}$. Na página inicial deste repositório, há um tutorial simples de como desenvolver um plugin e como adicioná-lo ao Portugol Studio.

O plugin é dividido em 3 partes que podem ser vistas na figura 2. As partes são: (i) Ações, que são equivalentes aos botões que serão adicionados à ferramenta pelo plugin. As ações são principalmente o que fará o plugin e onde o desenvolvedor trabalhará principalmente; (ii) Bibliotecas, que permite que novas bibliotecas sejam adicionadas à ferramenta; (iii) Licença, onde os autores devem colocar suas credenciais e descrição do seu plugin. 


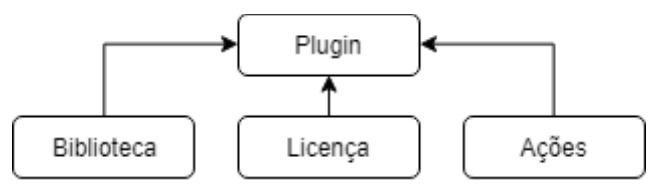

Figura 2. Arquitetura simplificada dos Plugins

Dependendo do tipo de aplicação que o usuário queira adicionar ao Portugol Studio, não será necessário um grande conhecimento de Java, entretanto como a maioria dos plugins devem fazer alterações na plataforma, será necessário o conhecimento de como o código fonte do Portugol Studio funciona. Neste caso é recomendado que se tente entender como a programação da plataforma funciona, baixando o código-fonte da IDE do repositório do Portugol Studio ${ }^{[2]}$.

\section{Ferramentas similares}

Além do Portugol Studio existem outras ferramentas similares voltadas a iniciantes em programação. A tabela 2 faz uma comparação breve destas ferramentas e seus recursos.

Tabela 2. Comparativo das IDEs similares ao Portugol Studio

\begin{tabular}{|l|c|c|c|c|c|}
\hline & $\begin{array}{c}\text { Portugol } \\
\text { Studio }\end{array}$ & VisualG & $\begin{array}{c}\text { Portugol } \\
\text { Viana }\end{array}$ & $\begin{array}{c}\text { Portugol } \\
\text { Online }\end{array}$ & $\begin{array}{c}\text { Portugol } \\
\text { IDE }\end{array}$ \\
\hline Depurador & Sim & Sim & Sim & Não & Sim \\
\hline Estrutura do programa & Sim & Não & Não & Não & Não \\
\hline Inspetor de variáveis & Sim & Sim & Sim & Não & Sim \\
\hline Fluxograma & Não & Não & Sim & Não & Sim \\
\hline Exemplos & Sim & Sim & Não & Não & Não \\
\hline Plugins & Sim & Não & Não & Não & Não \\
\hline Bibliotecas & Sim & Não & Não & Não & Não \\
\hline
\end{tabular}

\section{Perfil dos usuários}

A partir de 2017, o Portugol Studio iniciou em seu site ${ }^{[3]}$ uma coleta de dados sobre o perfil dos usuários que fazem download da ferramenta. Este capítulo tem como objetivo apresentar e discutir os dados coletados a fim de proporcionar uma melhor compreensão do perfil destes usuários. Os dados foram coletados no dia 20/03/2019 através do Google Analytics e são referentes ao período de 01/07/2017 a 19/03/2019.

Existem dois pontos importantes a serem observados na análise: (i) os dados não levam em conta possíveis usuários que já utilizavam o ambiente antes do início da coleta e; (ii) todas as estatísticas apresentadas têm como base o número de usuários (108.589), com exceção dos downloads que apresentam um número maior, uma vez que um mesmo usuário pode realizar vários downloads.

\subsection{Downloads}

Os dados a seguir, referem-se ao número de downloads do Portugol Studio. É importante observar que no passado a ferramenta era disponibilizada no SourceForge ${ }^{[4]}$ e se contabilizarmos os dados desta plataforma, o número aproximado será de 240.000 downloads. No entanto, nesta seção, serão considerados somente os dados coletados desde 2017 pelo Google Analytics. 


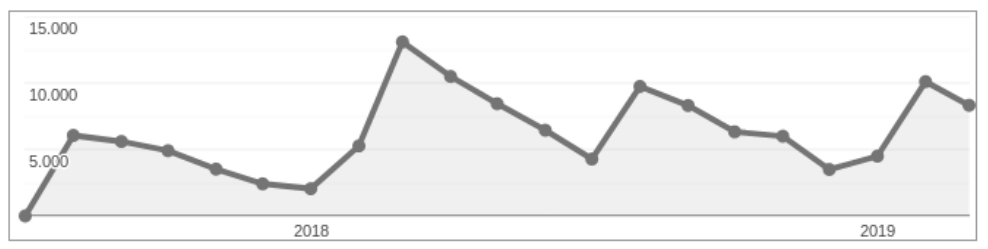

Gráfico 1. Número de downloads do Portugol Studio

Ao analisar o gráfico 1, pode-se observar que o Portugol Studio obteve um total de 129.277 downloads durante o período, o que equivale em média 6.156 downloads por mês. É possível ainda verificar o mínimo de 2.049 downloads em janeiro de 2018 (desconsiderando o mês em que a coleta foi iniciada) e o máximo de 13.081 downloads em março de 2018.

\subsection{Gênero e idade}

Nesta seção são apresentados dados estatísticos referentes ao gênero e à idade dos usuários. Estes dados foram calculados tomando como amostra apenas uma fração do número total de usuários (em torno de $50 \%$ ). Isto ocorre porque o Google Analytics não é capaz de coletar essas informações para todos os usuários.

Analisando os dados do gráfico 2, é possível verificar que quase metade dos usuários $(47,11 \%)$ se encontra na faixa etária entre 18 e 24 anos. Isto sustenta a hipótese de que boa parte do público alvo do Portugol Studio são alunos de graduação que estão ingressando em um curso na área de computação e/ou afins, uma vez que este perfil de usuário se encontra nesta faixa etária.

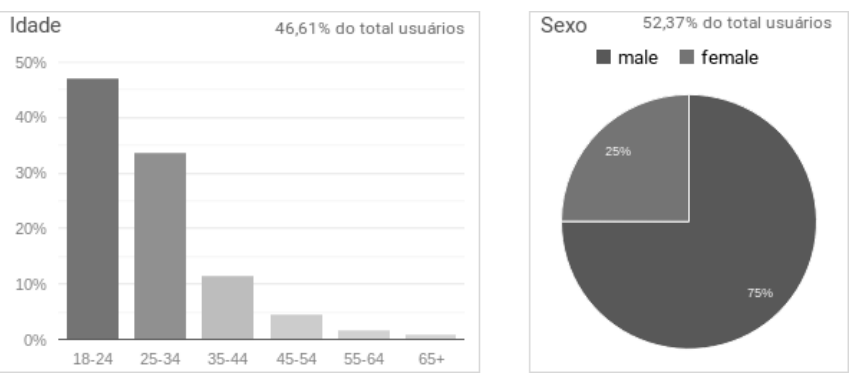

Gráfico 2. Gênero e idade dos usuários

Outras duas faixas etárias interessantes são 25 a 34 anos e 35 a 44 anos, correspondentes a $33,75 \%$ e $11,75 \%$ dos usuários. Juntas, estas duas faixas etárias compreendem $45,5 \%$ dos usuários e supõe-se que representam os professores de graduação que utilizam o Portugol Studio em suas aulas. Ainda referente à idade, podese notar que o restante dos usuários $(7,58 \%)$ está distribuído em diversas faixas etárias a partir dos 45 anos, demonstrando que talvez o Portugol Studio tenha potencial para atender públicos alvos de maior idade.

Já ao analisar os dados referentes ao gênero, é possível verificar que apenas $25 \%$ dos usuários são do gênero feminino, constatando o que muitas vezes é observado em sala de aula nos cursos de computação, onde o número de mulheres geralmente é menor. Esta informação é muito útil, pois aponta uma possível direção para a qual o Portugol Studio pode evoluir. No futuro, pode-se planejar formas de atrair e apoiar o público feminino. 


\subsection{Idiomas e nacionalidade}

Esta seção apresenta os dados relacionados ao idioma falado pelos usuários do Portugol Studio e países/regiões onde vivem. Em relação ao idioma, nesta análise considerou-se apenas os 10 primeiros idiomas registrados, os quais compreendem $99,78 \%$ dos usuários. Os demais $0,22 \%$ foram ignorados e estão distribuídos em 56 diferentes idiomas, a maioria representando menos de 5 usuários.

Ao analisar a tabela 2, percebe-se que a maior parte dos usuários fala o idioma Português, ou seja, este idioma compreende $93,73 \%$ dos usuários ao serem somados o Português Brasileiro, Europeu e demais dialetos. Já era esperado que o Português fosse o idioma dominante, uma vez que a ferramenta é voltada para o público falante desta língua.

Embora o idioma Português seja predominante, é possível perceber ao analisar a tabela 2, que os outros $6,05 \%$ dos usuários se distribuem entre variações do Inglês e do Espanhol, sendo que, dentre estes, o Inglês é o idioma com maior percentual de ocorrência. Enquanto que a ocorrência do idioma Espanhol é compreensível dadas as suas similaridades com Português, a ocorrência do Inglês por sua vez é bastante curiosa

Tabela 2. Idioma dos usuários do Portugol Studio

\begin{tabular}{|r|l|r|r|}
\hline & & 108.589 & 108.589 \\
10056 de 108.589 \\
\hline 1. & Português Brasileiro & 97.216 & $89,75 \%$ \\
\hline 2. & Inglês Americano & 5.632 & $5,20 \%$ \\
\hline 3. & Português Europeu (como escrito e falado em Portugal) & 4.027 & $3,72 \%$ \\
\hline 4. & Não identificado & 345 & $0,32 \%$ \\
\hline 5. & Demais variações/dialetos de Português & 282 & $0,26 \%$ \\
\hline 6. & Inglês Britânico & 241 & $0,22 \%$ \\
\hline 7. & Demais variações/dialetos do Inglês & 183 & $0,17 \%$ \\
\hline 8. & Espanhol Castelhano (como falado no centro-norte da Espanha) & 71 & $0,07 \%$ \\
\hline 9. & Inglês Canadense & 48 & $0,04 \%$ \\
\hline 10. & Demais variações/dialetos do Espanhol & 33 & $0,03 \%$ \\
\hline
\end{tabular}

Em relação à nacionalidade, ao analisar a tabela 3, os dados mostram que o Portugol Studio não foi utilizado apenas em países que falam o Português como língua nativa, mas também em outros países como Estados Unidos, Japão e Alemanha. Ao detalhar os acessos dos Estados Unidos, é possível verificar que as regiões do país em que o Portugol Studio foi baixado coincidem com os 12 estados com maior incidência de imigrantes, segundo dados publicados no site Lattice Publishing ${ }^{[5]}$.

Tabela 3. País e região de origem dos usuários do Portugol Studio

\begin{tabular}{|c|c|c|c|c|c|c|c|}
\hline & & $\begin{array}{r}108.589 \\
100 \% \text { de } 108.589\end{array}$ & $\begin{array}{r}108.589 \\
100 \% \text { de } 108.589\end{array}$ & & & $\begin{array}{r}424 \\
0,39 \% \text { de } 108.589\end{array}$ & $\begin{array}{r}424 \\
0,39 \% \text { de } 108.589\end{array}$ \\
\hline 1. & Brasil & 104.554 & $96,21 \%$ & 1. & Não identificado & 131 & $30,61 \%$ \\
\hline 2. & Portugal & 2.121 & | 1,95\% & 2. & California & 76 & $17,76 \%$ \\
\hline 3. & Angola & 575 & $0,53 \%$ & 3. & New Jersey & 26 & $6,07 \%$ \\
\hline 4. & Estados Unidos & 424 & $0,39 \%$ & 4. & Virginia & 25 & $5,84 \%$ \\
\hline 5. & Não identificado & 156 & $0,14 \%$ & 5. & New York & 24 & $5,61 \%$ \\
\hline 6. & E Moçambique & 113 & $0,10 \%$ & 6. & Texas & 19 & $4,44 \%$ \\
\hline 7. & Cabo Verde & 73 & $0,07 \%$ & 7. & Illinois & 17 & $3,97 \%$ \\
\hline 8. & • Japão & 67 & $0,06 \%$ & 8. & Florida & 16 & $3,74 \%$ \\
\hline 9. & 『»】 Canadá & 59 & $0,05 \%$ & 9. & Massachusetts & 13 & $3,04 \%$ \\
\hline 10. & 回 Alemanha & 56 & $0,05 \%$ & 10. & Utah & & | $2,10 \%$ \\
\hline
\end{tabular}

\section{Contribuições da comunidade}

Esta seção descreve brevemente as contribuições realizadas ao Portugol Studio pelos usuários da ferramenta e por trabalhos acadêmicos relacionados desenvolvidos com base no Portugol Studio. 


\subsection{Portugol WebStudio}

O Portugol WebStudio é um ambiente online que surgiu como uma adaptação do Portugol Studio devido à necessidade de torná-lo acessível em qualquer lugar. Seu objetivo é tentar trazer todos os recursos do ambiente desktop para a Internet. O projeto é uma iniciativa própria e é desenvolvido e mantido por Douglas Gadelha e Guilherme Scaranse ${ }^{[6]}$.

\subsection{Copa Rio Info de Algoritmos}

A Copa Rio Info de Algoritmos (CRIA) é uma competição de algoritmos que ocorre anualmente desde 2013 e utiliza o Portugol Studio como ambiente para a resolução dos problemas $^{[7]}$. É organizada pela TI Rio ${ }^{[8]}$ e Fenainfo ${ }^{[9]}$ na cidade do Rio de Janeiro. A CRIA visa atrair e estimular jovens talentos do ensino médio/técnico a buscar uma carreira na área das Tecnologias da Informação e Comunicação - TICs. Escolas de todo o Brasil, são convidadas participar da competição.

\subsection{Materiais instrucionais}

Pessoas na comunidade ajudaram independentemente na criação de diversos cursos de aprendizado de programação relacionados ao Portugol. Alguns deles são totalmente de graça disponiveis através do Youtube para qualquer pessoa. Abaixo estão alguns conteúdos que foram descobertos.

- Canais do Youtube: HM Programming ${ }^{[10]}$; Jean Vargas ${ }^{[11]}$; Nilson Júnior ${ }^{[12]}$; Prof RMuniz ${ }^{[13]}$

- Cursos de lógica de programação: Udemy - Programação para Iniciantes usando Portugol Studio ${ }^{[14]}$; Udemy - Introdução à Lógica de Programação com Portugol Studio $^{[15]}$; Timtec - Introdução à lógica de programação ${ }^{[16]}$

- Livros: Portugol Studio - Programação de computadores em Português - Guia de referência escolar ${ }^{[17]}$

\section{Considerações Finais}

A IDE Portugol Studio é bem vista pela comunidade. A simplicidade e facilidade de uso dela, faz com que seja ótima para que iniciantes comecem a programar sem a necessidade de entrar no mundo profissional diretamente. As constantes atualizações e o contato com o público da ferramenta, fez com que ela evoluísse rapidamente, trazendo novos recursos e corrigindo os problemas que ocorressem no processo.

A aceitação aumentou o número de usuários, o que permitiu coletar dados sobre o perfil deles. O perfil de usuário mais comum foi de homem, entre 18 e 24 anos brasileiro. Nos cursos de Ciência da Computação, este perfil é bem comum, pois são alunos que saíram a pouco tempo do ensino médio e querem aprender a programar.

O Portugol Studio é uma ferramenta de código aberto. Isso permite que a comunidade também possa dar contribuições a ela evoluindo-a ainda mais. Pesquisadores que queiram fazer seus projetos, podem pegar o código fonte da ferramenta e criar suas próprias modificações com base no Portugol Studio, alinhando com sua pesquisa. A existência dos plugins facilita ainda mais o processo quando ela também dá acesso aos recursos do Portugol Studio, porém sem a necessidade de realizar atualizações onde qualquer versão (a partir da 2.7 ) pode incorporar o plugin desenvolvido. 
Projetos que podem ser feitos com os recursos do Portugol Studio incluem: Sistemas Tutores Inteligentes, programação em blocos, tradutores de outras linguagens de programação, programação web, ferramentas inclusivas, entre outras. Além disso, a equipe do Portugol Studio está também disposta a compartilhar os dados que contém de uso da ferramenta para futuras pesquisas que possam ser feitas com eles.

Dentro da Univali ainda estão sendo feitas pesquisas relacionadas à ferramenta. Possibilidades de criar um caminho de aprendizado para o aluno quando inicia em programação, as dificuldades que o aluno tem para programar e a conexão internacional de pessoas pela programação, são temas pesquisados dentro do laboratório LITE. Com isso a ferramenta tende a continuar a evoluir com as pesquisas e a comunidade.
[1] https://bit.ly/30g1x9C
[7] https://bit.ly/2vTeUOO
[13] https://bit.ly/2E5LO3h
[2] https://bit.ly/2JB7acD
[8] https://bit.ly/2HdY3N7
[14] https://bit.ly/2vTXq5e
[3] https://bit.ly/2HhrwEW
[9] https://bit.ly/2WFhx2w
[10] https://bit.ly/2JgEari
[15] https://bit.ly/2NnYq7F
[4] https://bit.ly/1mHlgrn
[11] https://bit.ly/2vSScXp
[16] https://bit.ly/2HlXvUe
[5] https://bit.ly/2JcjTCY
[12] https://bit.ly/2Yi71ii
[17] https://bit.ly/2Jz49cM

\section{Referências}

Blikstein, P. (2014) "Bifocal Modeling: Promoting Authentic Scientific Inquiry Through Exploring and Comparing Real and Ideal Systems", In: Playful User Interfaces: Interfaces that Invite Social and Physical Interaction.

Breda, D. (2017) "Biblioteca para simulações de experimentos naturais", In: Trabalho Técnico de Conclusão de Curso, Ciência da Computação, UNIVALI - Universidade do Vale do Itajaí.

Cardoso, A. (2017) "Extensão do Portugol Studio para a controladora Gogo Board", In: Trabalho Técnico de Conclusão de Curso, Ciência da Computação, UNIVALI Universidade do Vale do Itajaí.

Sipitakiat, A. Blikstein, P. e Cavallo, D. P. (2004) "GoGo board: augmenting programmable bricks for economically challenged audiences", In: Proceedings of the 6th international conference on Learning sciences, pages 481-488.

Noschang, L. F., Pelz, F., Jesus, E. A., Raabe, A. L. A. (2014) "Portugol Studio: Uma IDE para Iniciantes em Programação", In: XXXIV Congresso da Sociedade Brasileira de Computação - CSBC 2014. 